\title{
(2) \\ (อ) OPEN ACCESS \\ Accuracy of a no-biopsy approach for the diagnosis of coeliac disease across different adult cohorts
}

\author{
Hugo A Penny (10, ${ }^{1}$ Suneil A Raju, ${ }^{1}$ Michelle S Lau, ${ }^{1}$ Lauren JS Marks, ${ }^{1}$ \\ Elisabeth MR Baggus, ${ }^{1}$ Julio C Bai, ${ }^{2}$ Gabrio Bassotti, ${ }^{3}$ Hetty J Bontkes, ${ }^{4}$ \\ Antonio Carroccio @ $1{ }^{5}$ Mihai Danciu, ${ }^{6}$ Mohammad H Derakhshan, ${ }^{7}$ Arzu Ensari, ${ }^{8}$ \\ Azita Ganjii, ${ }^{9}$ Peter H R Green, ${ }^{10}$ Matt W Johnson, ${ }^{11}$ Sauid Ishaq (10,12 \\ Benjamin Lebwohl, ${ }^{10}$ Adam Levene, ${ }^{11}$ Roxana Maxim, ${ }^{13}$ \\ Hamid Mohaghegh Shalmani, ${ }^{14}$ Mohammad Rostami-Nejad, ${ }^{14}$ David Rowlands, ${ }^{15}$ \\ Irene A Spiridon, ${ }^{6}$ Amitabh Srivastava $10,{ }^{16}$ Umberto Volta, ${ }_{1}^{17}$ Vincenzo Villanacci, ${ }^{18}$ \\ Graeme Wild, ${ }^{1}$ Simon S Cross, ${ }^{1}$ Kamran Rostami, ${ }^{19}$ David S Sanders ${ }^{1}$
}

For numbered affiliations see end of article.

\section{Correspondence to} Professor David S Sanders, Academic Unit of Gastroenterology, Royal Hallamshire Hospital, Sheffield S10 2JF, UK:

david.sanders1@nhs.net

Received 15 February 2020 Revised 29 June 2020 Accepted 18 July 2020 Published Online First 2 November 2020
Check for updates

(C) Author(s) (or their employer(s)) 2021. Re-use permitted under CC BY-NC. No commercial re-use. See rights and permissions. Published by BMJ.

To cite: Penny HA, Raju SA, Lau MS, et al. Gut

$2021: 70: 876-883$

\section{ABSTRACT}

Objective We aimed to determine the predictive capacity and diagnostic yield of a 10-fold increase in serum IgA antitissue transglutaminase (tTG) antibody levels for detecting small intestinal injury diagnostic of coeliac disease (CD) in adult patients.

Design The study comprised three adult cohorts. Cohort 1: 740 patients assessed in the specialist CD clinic at a UK centre; cohort 2: 532 patients with low suspicion for CD referred for upper GI endoscopy at a UK centre; cohort 3: 145 patients with raised tTG titres from multiple international sites. Marsh 3 histology was used as a reference standard against which we determined the performance characteristics of an IgA tTG titre of $\geq 10 \times U L N$ for a diagnosis of CD.

Results Cohort 1: the sensitivity, specificity, positive predictive value (PPV) and negative predictive value (NPV) for IgA tTG levels of $\geq 10 \times U L N$ at identifying individuals with Marsh 3 lesions were $54.0 \%, 90.0 \%$, $98.7 \%$ and $12.5 \%$, respectively. Cohort 2: the sensitivity, specificity, PPV and NPV for IgA tTG levels of $\geq 10 \times U L N$ at identifying individuals with Marsh 3 lesions were $50.0 \%, 100.0 \%, 100.0 \%$ and $98.3 \%$, respectively. Cohort 3: the sensitivity, specificity, PPV and NPV for IgA tTG levels of $\geq 10 \times U L N$ at identifying individuals with Marsh 3 lesions were $30.0 \%, 83.0 \%, 95.2 \%$ and $9.5 \%$, respectively.

Conclusion Our results show that IgA tTG titres of $\geq 10 \times U$ LN have a strong predictive value at identifying adults with intestinal changes diagnostic of CD. This study supports the use of a no-biopsy approach for the diagnosis of adult CD.

\section{INTRODUCTION}

Coeliac disease (CD) is a common immunemediated enteropathy, whereby increased immunological sensitivity to dietary gluten results in chronic small intestinal mucosal inflammation in genetically susceptible individuals. ${ }^{1}$ CD affects $\sim 1 \%$ of the global population, but despite its rising prevalence, the majority of patients remain undiagnosed. ${ }^{2}$ Currently, most patients with suspected CD

\section{Significance of this study}

What is already known on this subject?

- Paediatric European Society for the Study of Paediatric Gastroenterology, Hepatology and Nutrition guidelines suggest that a diagnosis of coeliac disease (CD) can be made without taking duodenal biopsies.

- The latest criteria suggest that a 10-fold increase in $\lg \mathrm{A}$ antitissue transglutaminase (tTG) antibody levels in combination with EMA positivity is sufficient to make a diagnosis of $C D$ in the absence of duodenal biopsies.

- In adults, this approach has not yet been widely adopted into clinical practice, largely due to a lack of international multicentre data and testing in low CD prevalence cohorts.

What are the new findings?

- Across three cohorts of adult patients, we have shown that almost all individuals with IgA tTG titres of $\geq 10 \times$ ULN have small intestinal mucosal changes diagnostic of CD (Marsh 3 lesions) on duodenal biopsy.

- We show that IgA tTG titres of $10 \times$ ULN have $100 \%$ specificity at detecting Marsh 3 lesions in a cohort of 532 adults with a CD prevalence of $3.2 \%$.

- Finally, we found that an IgA tTG cut-off of $10 \times$ ULN performed well at identifying individuals with Marsh 3 lesions using different assays across multiple international sites. However, determining assay-specific thresholds and/or standardisation of tTG assays used with this pathway may help to optimise the accuracy and impact of this approach.

How might it impact on clinical practice in the foreseeable future?

- This study supports a change in guidelines towards a no-biopsy approach for the diagnosis of $C D$ within adult gastroenterology services. 
are screened serologically for antibody positivity, in particular for IgA antibodies to tissue transglutaminase 2 (tTG) and antiendomysial IgA antibodies (EMAs). ${ }^{1}$ As these serological markers are not $100 \%$ specific for detecting intestinal lesions compatible with CD, positive coeliac serology is confirmed by duodenal biopsies demonstrating the hallmark pathological changes of mucosal remodelling, such as villous atrophy, crypt hyperplasia and intraepithelial lymphocytosis. ${ }^{3}$

However, upper GI endoscopy with biopsies is expensive, with costs relating directly to the procedure and indirectly to the processing and analysing of samples. The procedure is poorly tolerated by many patients ${ }^{4}$ and carries risks, including sedation complications and although rare in current practice, GI haemorrhage and/or perforation. ${ }^{5}$ Furthermore, variability exists in the identification of the main histological changes of $\mathrm{CD}$, meaning cases can be missed despite duodenal biopsy sampling. ${ }^{67}$ Therefore, the identification of a non-invasive approach to the diagnosis of $\mathrm{CD}$ is of great interest.

The 2012 European Society for the Study of Paediatric Gastroenterology, Hepatology and Nutrition (ESPGHAN) guidelines for the diagnosis of $\mathrm{CD}$ challenged the necessity for duodenal biopsies in paediatric patients. They suggested that an IgA tTG antibody titre greater than 10 times the upper limit of normal (ULN), in combination with a positive EMA antibody test and compatible human leucocyte antigen (HLA) genotype, is sufficient to support a diagnosis of $\mathrm{CD}$ in symptomatic individuals. ${ }^{8}$ This eliminates the need for gastroscopy and its associated costs/risks in selected paediatric patients. ${ }^{8}$ The latest guidelines published this year remove the requirement for the presence of symptoms and HLA testing in the diagnostic pathway. ${ }^{9}$ This underscores the specificity of a serologybased or 'no-biopsy' approach for the diagnosis of paediatric CD.

Studies have evaluated whether this strategy can be applied in symptomatic adult $\mathrm{CD} .{ }^{10-21}$ These studies have suggested that $\mathrm{tTG}$ levels of $\geq 10 \times \mathrm{ULN}$ could be predictive of CD in adults, and the recently published Finnish national guidelines for the diagnosis of $\mathrm{CD}$ have incorporated this diagnostic pathway into their practice. ${ }^{22}$ However, this approach has not been widely adopted into adult clinical practice or guidelines. Adult gastroenterologists have cited the lack of international, multicentre data including testing in low CD prevalence populations and thus intimated caution. ${ }^{23}$ Furthermore, there are inconsistencies in the histological diagnosis of $\mathrm{CD}$ in the published literature. ${ }^{23}$ Therefore, the aim of the current study was to assess whether a tTG level of $\geq 10 \times \mathrm{ULN}$ could be sufficient for detecting duodenal mucosal abnormalities diagnostic of $\mathrm{CD}$ and to determine the diagnostic yield of this approach across different adult cohorts. In doing so, we aimed to address the shortcomings within the adult literature.

\section{METHODS}

\section{Patients}

The study comprised three adult ( $\geq 16$ years old) cohorts; all individuals included in the study underwent clinical assessment and had IgA tTG serology testing alongside upper GI endoscopy with duodenal biopsies performed.

Cohort 1 comprised a prospective analysis of consecutive adult patients assessed in the specialist CD clinic Royal Hallamshire Hospital, UK, from 2008 to 2019 who had a serum IgA tTG test and duodenal biopsy within 6 weeks of each other, no previous diagnosis of $\mathrm{CD}$, naivety to a gluten-free $\operatorname{diet}(\mathrm{GFD})$ and IgA competency. As these individuals were assessed within the specialist coeliac clinic, they were considered as having high clinical suspicion for CD.
Cohort 2 comprised a retrospective analysis of a prospectively recruited (December 2014-January 2017) ${ }^{24}$ cohort of adults referred for upper GI endoscopy at the Royal Hallamshire Hospital, UK. Patients were recruited prospectively from an endoscopy list that is for the investigation of GI complaints, as well as general and open-access referrals. Those with IgA deficiency, with a previous diagnosis of $\mathrm{CD}$ and on gluten restriction prior to diagnosis were excluded from analysis. To reduce the referral bias for CD, patients referred with positive coeliac serology (EMA positivity) in primary care were also excluded from the analysis. As a result, we considered this cohort as having low suspicion for $\mathrm{CD}$.

Cohort 3 comprised a retrospective analysis of adult patients with raised tTG titres from multiple sites, involving 8 countries and comprising 11 laboratories. Cases were identified based on positive coeliac serology and having had appropriate duodenal biopsy sampling. The eight countries included Argentina $(n=11)$, Iran $(n=10)$, Netherlands $(n=10)$, Italy $(n=44)$, Romania $(n=10)$, Turkey $(n=10)$, the USA $(n=40)$ and the UK $(n=10)$. In total, this provided assessments on 145 patients.

\section{Serology and biopsy}

All patients from cohorts 1 and 2 underwent serological testing and duodenal biopsy within 6 weeks of each other at the Royal Hallamshire Hospital in the context of a routine clinical service, with the exception that histopathologists were blinded to the serological status of patients from cohort 2. IgA tTG antibody levels were measured by ELISA using the Aeskulisa Diagnostics (Wendelsheim, Germany) kit before December 2014 and the ELiA Celikey (Thermo Fisher, Freiburg, Germany) after December 2014. Results were reported in unit per millilitre using the manufacturer's supplied reference ranges: $0-15$ negative, $>15$ positive, $0-7$ negative and $>7$ positive, respectively. The highest standard in the assay is 300 and $128 \mathrm{U} / \mathrm{mL}$, respectively, and these were used as upper limits for analysis, irrespective of whether optical density values exceeded this. IgA EMA was detected by immunofluorescence on primate oesophagus sections (Binding Site, Birmingham, UK).

During gastroscopy, at least four quadrantic biopsies were taken from the second part of the duodenum (D2) and one from the duodenal bulb using the single bite technique $(21 \%$ of patients from cohort 1 had $>1$ D2 biopsy only). The histopathology department at this site had significant experience in CD diagnosis. Duodenal mucosal changes were assessed using the Marsh criteria; Marsh 3 lesions were classified based on evidence of increased intraepithelial lymphocytes, crypt hyperplasia and villous atrophy. ${ }^{325}$ The most severe histological grade detected on biopsy was recorded and used for analysis. Unless otherwise stated, a diagnosis of typical CD was based on positive coeliac serology and Marsh 3 histology on duodenal biopsy. Seronegative CD was diagnosed in individuals with Marsh 3 lesions and supporting $\mathrm{CD}$ risk factors and/or response to a GFD; non-coeliac causes of villous atrophy were ruled out in these individuals.

Individuals from cohort 3 had IgA tTG titres measured by ELISA assays in routine clinical use at each site, including ARUP Laboratories (Utah, USA), QuantaLite (Inova Diagnostics, San Diego, California), Eu-tTG (Eurospital, Italy), Euroimmune (Luebeck, Germany) and ELiA Celikey (Thermo Fisher, Freiburg, Germany). Each individual had four to six duodenal biopsies sampled during upper GI endoscopy. A diagnosis of $\mathrm{CD}$ was made locally by expert gastroenterologists taking into consideration serology and duodenal biopsy results. 


\begin{tabular}{lc}
\hline & Cohort 1 \\
\hline$n$ & 740 \\
\hline Median age, years (IQR) & $42(27-55)$ \\
\hline Female:male & $492: 248$ \\
\hline Main presenting symptoms & $\%(n)$ \\
• Gastrointestinal & $41 \%(302)$ \\
• Extra-intestinal & $9 \%(70)$ \\
- Malabsorptive & $42 \%(314)$ \\
- Asymptomatic & $2 \%(13)$ \\
- No details & $6 \%(41)$ \\
\hline Marsh scores & $\%(n)$ \\
- 0 & $2 \%(11)$ \\
- $1 \& 2$ & $5 \%(39)$ \\
\hline Coeliac disease diagnosis & $93 \%(690)$ \\
• Typical & $\%(n)$ \\
- Seronegative & $90 \%(668)$ \\
\hline
\end{tabular}

B

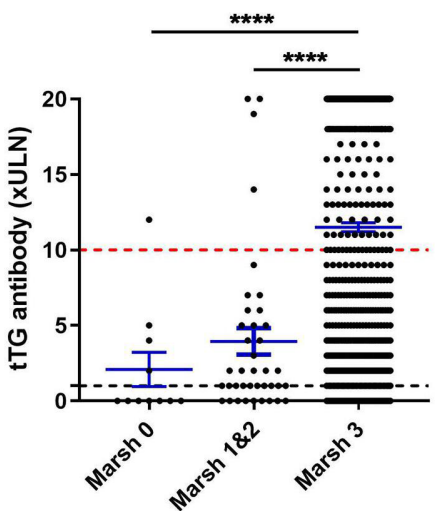

C

\begin{tabular}{lcc}
\hline & \multicolumn{2}{c}{$\geq \mathbf{1 0 x}$ ULN } \\
\cline { 2 - 3 } & Value & $\mathbf{9 5 \%} \mathbf{C l}$ \\
\hline Sensitivity & $54 \%$ & $51-58 \%$ \\
\hline Specificity & $90 \%$ & $78-97 \%$ \\
\hline PPV & $98.7 \%$ & $97.0-99.4 \%$ \\
\hline NPV & $12.5 \%$ & $11.2-13.9 \%$ \\
\hline
\end{tabular}

Figure 1 (A) Demographic and clinical details of the patients in cohort 1. Gl symptoms include abdominal pain, dyspepsia, dysphagia, nausea, vomiting, bloating and constipation; extraintestinal symptoms include ataxia, headache, dizziness, fatigue, joint pain and skin rash; malabsorptive symptoms include diarrhoea, haematinic deficiencies with/without anaemia and weight loss. (B) Normalised tTG titres against Marsh grade in patients from cohort 1. Horizontal bar represents the mean; error bars denote SE of the mean. Black dotted line represents $1 \times U L N$ threshold; red dotted line represents $10 \times$ ULN threshold. Comparisons between groups made with Kruskal-Wallis and post hoc Dunn test. (C) Performance characteristics of tTG titres of $10 \times$ ULN at predicting villous atrophy. ${ }^{* * *} \mathrm{P}<0.0001$. $\mathrm{tTG}$, tissue transglutaminase; ULN, upper limit of normal; PPV, positive predictive value; NPV, negative predictive value.

\section{Ethical consideration}

All identifiable medical information was removed, and all analyses were performed using anonymised data. See below for full details.

\section{Statistical analysis}

Data handling was conducted using spreadsheets in Microsoft Excel (2016); statistical analysis and graph construction were performed using GraphPad Prism V.7 (GraphPad Software, San Diego, California, USA). To enable comparison of tTG levels between different assays, tTG levels were normalised by expression as a multiple of the ULN-defined as the lowest value that was considered abnormal for each assay. ${ }^{19}$ Where indicated, receiver operating characteristic (ROC) curves were constructed, and area under the curve (AUC) was calculated to determine the accuracy of tTG levels at predicting villous atrophy. Analysis of variance or Kruskal-Wallis test, with appropriate post hoc analysis, was used to compare Marsh grades with tTG titres. A p value of $<0.05$ was considered statistically significant.

\section{RESULTS}

Cohort 1: patients assessed in the specialist CD clinic

A total of 740 patients fulfilled the criteria and were included in cohort 1 . The prevalence of CD in this cohort was $93 \%$ (690/740 patients). Patient demographics and clinical details are listed in figure 1A. Altogether, 380/740 (51\%) had tTG titres of $\geq 10 \times$ ULN. Marsh 3 lesions were detected in 375/380 (98.7\%) of those with tTG titres of $\geq 10 \times \mathrm{ULN}$ (figure $1 \mathrm{~B}$ ). Of the 740 patients, $360(49 \%)$ had tTG titres of $<10 \times \mathrm{ULN}$; of those, 315/360 (87.5\%) had Marsh 3 lesions, 35/360 (9.7\%) had Marsh 1-2 lesions and 10/360 (2.8\%) had Marsh 0 lesions on duodenal biopsy. Based on this, the positive predictive value (PPV) of tTG values of $\geq 10 \times \mathrm{ULN}$ at predicting mucosal abnormalities with villous atrophy was 98.7\% (97.0\%-99.4\%) (figure 1C). Five patients had tTG titres of $\geq 10 \times \mathrm{ULN}$ without detectable villous abnormalities (ie, Marsh $<3$ lesions); the demographic and clinical characteristics of these patients are shown in table 1. All five patients had symptoms of malabsorption and/or were EMA positive and all were positive for the HLA DQ2/8 genotype. All patients were started on a GFD and subsequently, tTG titres decreased.

Two different tTG assays were used to test tTG titres in cohort 1 over the study period; we next evaluated the differences between these two assays. To do this, we divided the cohort into two, based on the tTG assay used (cohort 1A, Aeskulisa Diagnostics; cohort 1B, ELiA Celikey). The demographics of individuals included in cohort $1 \mathrm{~A}$ and $1 \mathrm{~B}$ are detailed in figure $2 \mathrm{~A}$. Analysis of tTG titres showed that there were differences in the predictive power of the two tests, such that applying a $10 \times$ ULN cut-off had a PPV of 98.1\% (95.7\%-99.1\%) for Marsh 3 lesions in cohort

Table 1 Demographic and clinical details of patients with tTG titres $\geq 10 \times$ ULN where villous abnormalities were not detected on duodenal biopsies

\begin{tabular}{|c|c|c|c|c|c|}
\hline & Patient 1 & Patient 2 & Patient 3 & Patient 4 & Patient 5 \\
\hline Age (years) & 34 & 42 & 43 & 33 & 46 \\
\hline Gender & Female & Male & Female & Male & Female \\
\hline Presenting symptoms & Diarrhoea with urgency & Diarrhoea, abdominal pain & Iron deficiency anaemia, diarrhoea & Abdominal pain, bloating, tiredness & Iron deficiency anaemia \\
\hline EMA test & Negative & Positive & Negative & Positive & Negative \\
\hline Family history of $\mathrm{CD}$ ? & No & No & No & No & No \\
\hline TTG post-GFD & 1 & 8 & 6 & 111 & 1 \\
\hline
\end{tabular}

CD, coeliac disease; EMA, endomysial antibody; GFD, gluten free diet; HLA, human leucocyte antigen; tTG, tissue transglutaminase. 
A

\begin{tabular}{lcc}
\hline & Cohort 1A & Cohort 1B \\
\hline$n$ & 467 & 273 \\
\hline Median age, years (IQR) & $43(28-55)$ & $42(26-56)$ \\
\hline Female:male & $311: 156$ & $181: 92$ \\
\hline Marsh 0 & 6 & 5 \\
\hline Marsh 1 \& & 28 & 11 \\
\hline Marsh 3 & 433 & 257 \\
\hline
\end{tabular}
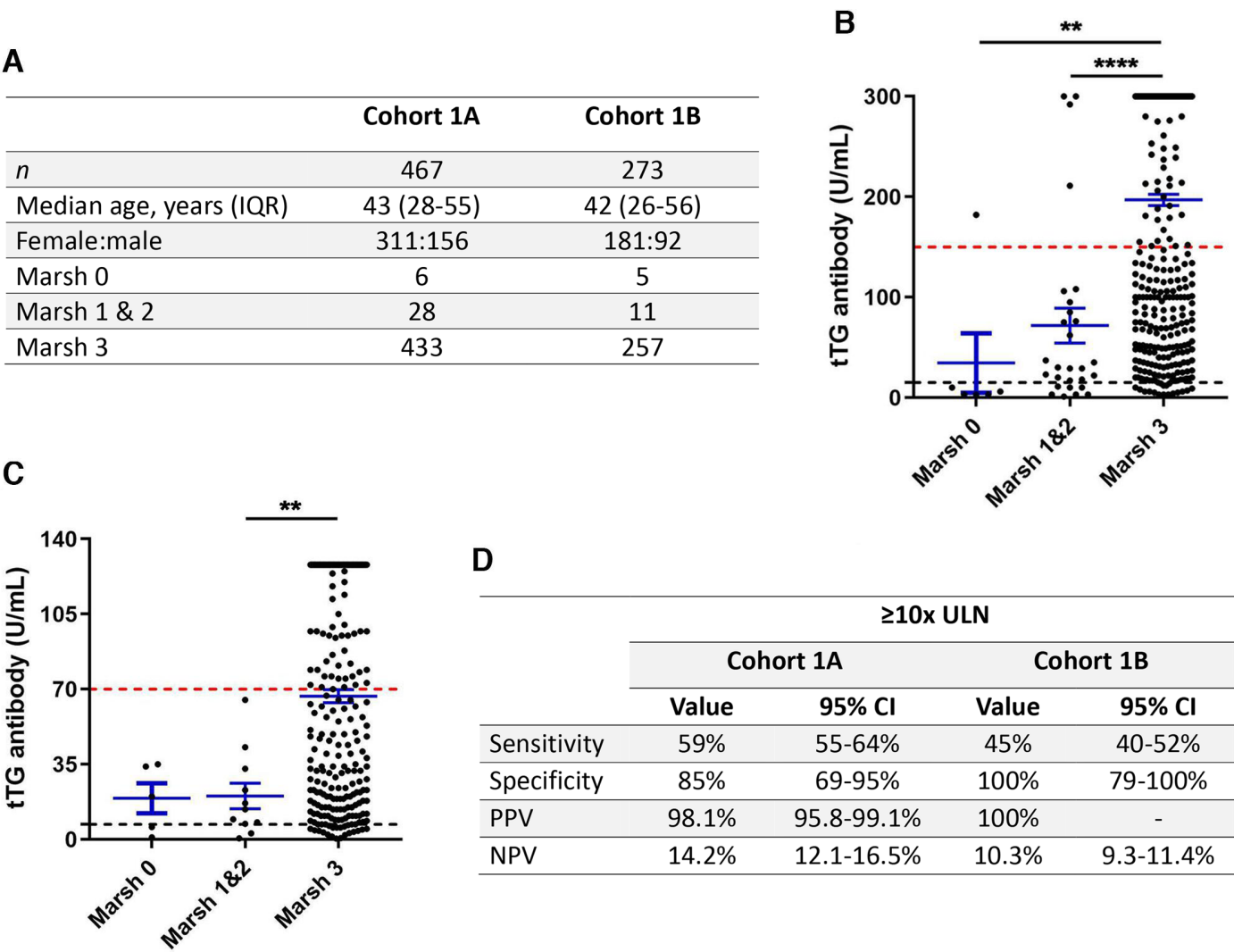

D

\begin{tabular}{lcccc}
\hline & \multicolumn{4}{c}{$\geq 10 x$ ULN } \\
\cline { 2 - 5 } & \multicolumn{2}{c}{ Cohort 1A } & \multicolumn{2}{c}{ Cohort 1B } \\
\cline { 2 - 5 } & Value & $\mathbf{9 5 \% ~ C l}$ & Value & $\mathbf{9 5 \% ~ C l}$ \\
\hline Sensitivity & $59 \%$ & $55-64 \%$ & $45 \%$ & $40-52 \%$ \\
\hline Specificity & $85 \%$ & $69-95 \%$ & $100 \%$ & $79-100 \%$ \\
\hline PPV & $98.1 \%$ & $95.8-99.1 \%$ & $100 \%$ & - \\
\hline NPV & $14.2 \%$ & $12.1-16.5 \%$ & $10.3 \%$ & $9.3-11.4 \%$ \\
\hline
\end{tabular}

Figure 2 (A) Demographic and clinical details of the patients in cohorts 1A and 1B. (B) tTG titres against Marsh grade in patients from cohort 1A. (C) tTG titres against Marsh grade in patients from cohort 1B. Horizontal bar represents the mean; error bars denote SE of the mean. Black dotted line represents $1 \times U L N$ threshold; red dotted line represents $10 \times U L N$ threshold. Comparisons between groups were made with analysis of variance and post hoc Tukey test. (D) Performance characteristics of tTG titres of $10 \times \mathrm{ULN}$ at predicting villous atrophy. ${ }^{* *} \mathrm{P}<0.01,{ }^{* * * *} \mathrm{P}<0.0001$. tTG, tissue transglutaminase; ULN, upper limit of normal; PPV, positive predictive value; NPV, negative predictive value.

1A, but a PPV of $100 \%$ in cohort 1B (figure 2B-D). Notably, the very high tTG titres in patients with $\mathrm{Marsh}<3$ lesions were recorded using the Aeskulisa assay (figure 2B).

\section{Cohort 2: patients referred for upper GI endoscopy}

A total of 778 patients were included in cohort 2 . Ten patients had IgA deficiency; 91 patients were already consuming a GFD and/or had a diagnosis of CD; 1 patient had clinical features in keeping with a relapse of known small bowel Crohn's disease; and 23 individuals had incomplete testing or missing information, and so these individuals were excluded from further analysis. Of the remaining cohort, 121 patients had positive EMA testing in primary care, and so these were excluded from further analysis.

Therefore, 532 patients were included in the analysis stage. The prevalence of CD in this cohort was 3.2\% (17/532 patients). Patient demographics and clinical details are presented in figure 3A. 18/532 patients had Marsh 3 lesions identified on duodenal histology, 15 patients (2.8\% of the cohort) were diagnosed with typical CD; 2 patients $(0.4 \%$ of the cohort) had seronegative $\mathrm{CD}$; and 1 patient $(0.2 \%$ of the cohort) was diagnosed with non-coeliac seronegative villous atrophy.

The ELiA Celikey was used to measure tTG levels in all patients in cohort 2. Altogether, 9/532 (1.7\%) had tTG titres of $\geq 10 \times$ ULN. Marsh 3 lesions were detected in 9/9 (100\%) of those with tTG titres of $\geq 10 \times \mathrm{ULN}$ (figure $3 \mathrm{~B}$ ). All individuals (9/9) with tTG titres of $\geq 10 \times$ ULN were EMA positive. Of the 532 patients included, 523 (98.3\%) had tTG titres of
$<10 \times$ ULN; of those, 9/523 (1.7\%) had Marsh 3 lesions; 33/523 (6.3\%) had Marsh 1-2 lesions; and 481/523 (92.0\%) had Marsh 0 lesions on duodenal histology. Thus, the PPV of tTG values of $\geq 10 \times$ ULN at predicting individuals with Marsh 3 lesions was $100 \%$ (figure 3C).

ROC analysis showed an AUC of 0.9179 for tTG values at predicting Marsh 3 histology (figure 4A). Based on this analysis, the optimal threshold of tTG titres was $5.9 \times$ ULN (tTG titres of $>41.5 \mathrm{U} / \mathrm{mL}$ ) (figure $4 \mathrm{~B}$ ). Reducing the threshold to this level increased the sensitivity of this approach from $50 \%$ to $61 \%$. These results demonstrate that tTG titres of $10 \times \mathrm{ULN}$ have excellent specificity at detecting Marsh 3 lesions in a cohort of predominantly symptomatic adult patients with a low suspicion/prevalence of CD. Reducing the tTG threshold increased the number of individuals who would have benefited from this strategy, without compromising the predictive value of this approach.

\section{Cohort 3: international multicentre cohort}

A total of 145 patients were included in cohort 3. The prevalence of CD in this cohort was $92 \%$ (133/145 patients). Demographic and clinical characteristics are included in figure 5A. Of the 145 patients included, $42(29.0 \%)$ had tTG titres of $\geq 10 \times \mathrm{ULN}$, of which 40/42 (95.2\%) patients had Marsh 3 lesions on duodenal biopsy (figure 5B). One hundred three of the 145 patients (71.0\%) had tTG titres of $<10 \times \mathrm{ULN}$; of those, 93/103 (90.3\%) patients had Marsh 3 lesions; 6/103 (5.8\%) patients had Marsh 1-2 lesions; and 4/103 (3.9\%) had Marsh 0 lesions on duodenal 
A

\begin{tabular}{lc}
\hline & Cohort 2 \\
\hline$n$ & 532 \\
\hline Median age, years (IQR) & $57(44-70)$ \\
\hline Female:male & $327: 205$ \\
\hline Main presenting symptoms & $\%(n)$ \\
- Gastrointestinal & $66 \%(353)$ \\
- Extra-intestinal & $7 \%(37)$ \\
• Malabsorptive & $26 \%(136)$ \\
- Asymptomatic & $1 \%(6)$ \\
\hline Marsh scores & $\%(n)$ \\
• 0 & $91 \%(481)$ \\
• $1 \& 2$ & $6 \%(33)$ \\
\hline Coeliac disease diagnosis & $3 \%(18)$ \\
• Typical & $\%(n)$ \\
• Seronegative & $2.8 \%(15)$ \\
\hline
\end{tabular}

B

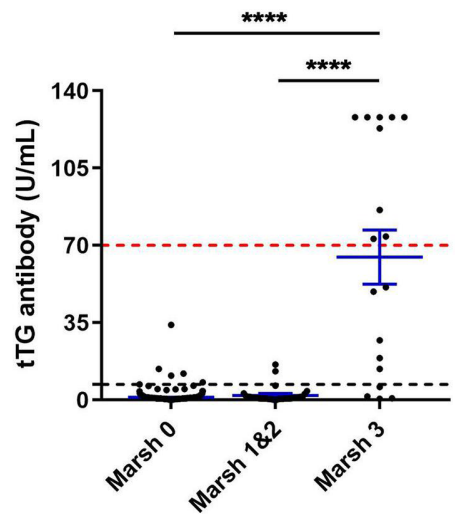

C

\begin{tabular}{lcc}
\hline & \multicolumn{2}{c}{$\geq \mathbf{1 0 x}$ ULN } \\
\cline { 2 - 3 } & Value & $\mathbf{9 5 \%} \mathbf{~ C l}$ \\
\hline Sensitivity & $50 \%$ & $\mathbf{2 6 - 7 4 \%}$ \\
\hline Specificity & $100 \%$ & $99-100 \%$ \\
\hline PPV & $100 \%$ & - \\
\hline NPV & $98.3 \%$ & $96.8-99.2 \%$ \\
\hline
\end{tabular}

Figure 3 (A) Demographic and clinical details of the patients in cohort 2. Gl symptoms include abdominal pain, dyspepsia, dysphagia, nausea, vomiting, bloating and constipation; extraintestinal symptoms include ataxia, headache, dizziness, fatigue, joint pain and skin rash; malabsorptive symptoms include diarrhoea, haematinic deficiencies with/without anaemia and weight loss. (B) tTG titres against Marsh grade in patients from cohort 2. Horizontal bar represents the mean; error bars denote SE of the mean. Black dotted line represents 1×ULN threshold; red dotted line represents 10xULN threshold. Comparisons between groups were made with analysis of variance and post hoc Tukey test. (C) Performance characteristics of tTG titres of $10 \times \mathrm{ULN}$ at predicting villous atrophy. ${ }^{* * *} \mathrm{P}<0.0001$. $\mathrm{tTG}$, tissue transglutaminase; ULN, upper limit of normal; PPV, positive predictive value; NPV, negative predictive value.

biopsy. Based on this, the predictive value of using a $10 \times$ ULN threshold at detecting individuals with Marsh 3 lesions was $95.2 \%(84.6 \%-98.7 \%)$ (figure 5C). Two patients that had tTG titres of $\geq 10 \times$ ULN without villous abnormalities both had Marsh 2 lesions on duodenal biopsy and were diagnosed with CD. Interestingly, tTG titres did not reach the $10 \times \mathrm{ULN}$ threshold across three sites, despite 56 individuals having Marsh 3 lesions across these sites. Therefore, a uniform tTG threshold of $10 \times \mathrm{ULN}$ may exclude many true positive cases.

\section{DISCUSSION}

Altogether this study included 1417 patients; 431/1417 (30\%) patients had tTG titres of $\geq 10 \times$ ULN, of which 424 (98\%) patients had Marsh 3 lesions on duodenal biopsy and were diagnosed with $\mathrm{CD}$. This study addresses the concerns raised by the adult gastroenterology community and supports a change in guidelines towards a no-biopsy approach for the diagnosis of $\mathrm{CD}$ in adults.

We identified Marsh 3 histology as our primary reference standard across all cohorts because (1) small intestinal mucosal remodelling, in keeping with Marsh 3 histology, represents the hallmark pathological changes in $\mathrm{CD}^{26}$; (2) few studies in the adult literature have reported on the predictive capacity of tTG titres of $\geq 10 \times$ ULN against Marsh 3 histology; rather, a clinical diagnosis of CD (based on the constellation of appropriate symptoms, serology and associated risk factors, in the absence of villous abnormalities) has been used as a reference standard by many ${ }^{27}$; and (3) few studies have included appropriate details regarding the location (including the duodenal bulb) and interpretation of biopsies. ${ }^{28}$ By contrast, our centre in Sheffield, UK, has published extensively on the matter of duodenal biopsy sampling in adult $\mathrm{CD} .{ }^{29-32}$

In taking this approach, initially, we evaluated the predictive value of tTG titres of $\geq 10 \times \mathrm{ULN}$ against the presence of Marsh 3 histology in patients seen in the specialist CD clinic (cohort 1). Most individuals within this cohort were diagnosed with $C D$, which reflects a high referral bias and pretest probability for $\mathrm{CD}$ in this cohort. This aligns with many previous studies, which have also assessed the diagnostic accuracy of tTG titre multiples of the ULN in high pretest probability/disease prevalence

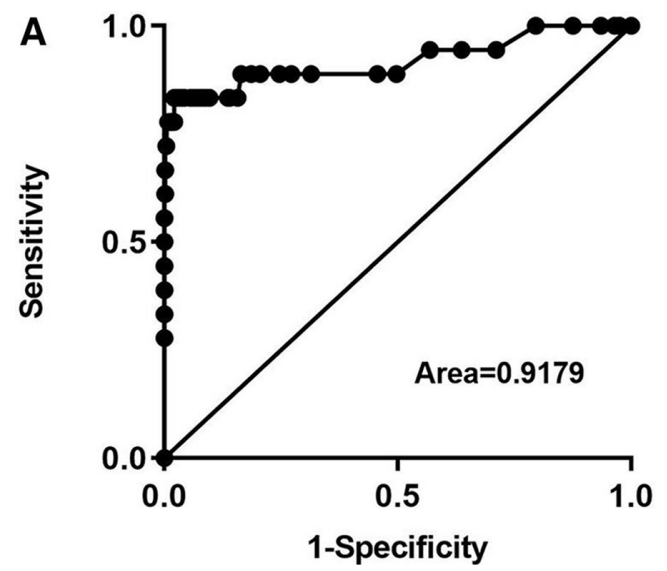

B

\begin{tabular}{lcc}
\hline & \multicolumn{2}{c}{$\geq 5.9 x$ ULN } \\
\cline { 2 - 3 } & Value & $\mathbf{9 5 \% ~ C l}$ \\
\hline Sensitivity & $61 \%$ & $36-83 \%$ \\
\hline Specificity & $100 \%$ & $99-100 \%$ \\
\hline PPV & $100 \%$ & - \\
\hline NPV & $98.7 \%$ & $97.6-99.2 \%$ \\
\hline
\end{tabular}

Figure 4 (A) Receiver operating characteristic curve analysis of tTG values against Marsh 3 histology in cohort 2. (B) Performance characteristics of tTG titres $\times 5.9$ ULN at predicting Marsh 3 lesions. tTG, tissue transglutaminase; ULN, upper limit of normal; PPV, positive predictive value; NPV, negative predictive value. 
A

\begin{tabular}{lc}
\hline & Cohort 3 \\
\hline$n$ & 145 \\
\hline Median age, years (IQR) & $36(26-50)$ \\
\hline Female:male & $111: 34$ \\
\hline Main presenting symptoms & $\%(n)$ \\
- Gastrointestinal & $15 \%(22)$ \\
- Extra-intestinal & $14 \%(20)$ \\
- Malabsorptive & $63 \%(91)$ \\
• Asymptomatic & $8 \%(11)$ \\
\hline Marsh scores & $\%(n)$ \\
- 0 & $3 \%(4)$ \\
- $1 \& 2$ & $5 \%(8)$ \\
Coeliac disease diagnosis & $92 \%(133)$ \\
• Typical & $\%(n)$ \\
• Seronegative & $92 \%(133)$ \\
\hline
\end{tabular}

B

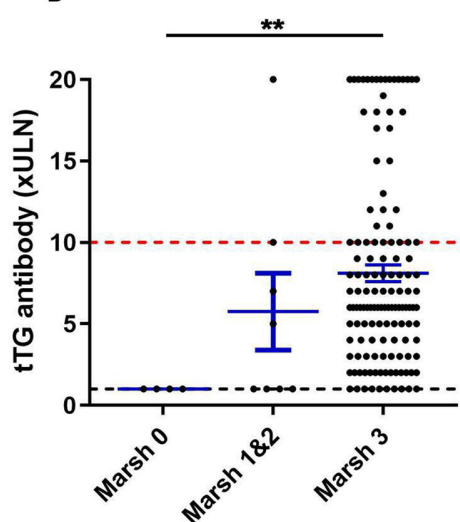

C

\begin{tabular}{lcc}
\hline & \multicolumn{2}{c}{$\geq \mathbf{1 0 x}$ ULN } \\
\cline { 2 - 3 } & Value & $\mathbf{9 5 \%} \mathbf{~ C I}$ \\
\hline Sensitivity & $30 \%$ & $22-38 \%$ \\
\hline Specificity & $83 \%$ & $52-98 \%$ \\
\hline PPV & $95.2 \%$ & $84.6-98.6 \%$ \\
\hline NPV & $9.5 \%$ & $7.4-12.2 \%$ \\
\hline
\end{tabular}

Figure 5 (A) Demographic and clinical details of the patients in cohort 3. Gl symptoms include abdominal pain, dyspepsia, dysphagia, nausea, vomiting, bloating and constipation; extraintestinal symptoms include ataxia, headache, dizziness, fatigue, joint pain and skin rash; malabsorptive symptoms include diarrhoea, haematinic deficiencies with/without anaemia and weight loss. (B) Normalised tTG titres against Marsh grade in patients from cohort 3. Horizontal bar represents the mean; error bars denote SE of the mean. Black dotted line represents $1 \times U$ LN threshold; red dotted line represents 10xULN threshold. Comparisons between groups made with Kruskal-Wallis and post hoc Dunn test. (C) Performance characteristics of tTG titres of $10 \times U L N$ at predicting villous atrophy. ${ }^{*} P<0.01$. $\mathrm{tTG}$, tissue transglutaminase; ULN, upper limit of normal; PPV, positive predictive value; NPV, negative predictive value.

populations (CD prevalence > 70\%). ${ }^{1012-141618}$ In accordance with these previous studies, we found that a tTG titre threshold of $10 \times \mathrm{ULN}$ accurately identified individuals with Marsh 3 lesions.

However, the pretest probability/disease prevalence within a cohort can influence the PPV of a diagnostic test. ${ }^{33}$ This means that as the disease prevalence increases, the more likely it is that a positive test represents a true positive case. ${ }^{33}$ The predictive value of the $10 \times$ ULN threshold in adults has less commonly been evaluated in more general clinical scenarios where the disease prevalence of CD is considered $<10 \% .{ }^{1128} 33$ Sugai et al previously demonstrated that a tTG titre threshold of around $7 \times$ ULN had a $100 \%$ PPV for CD in an adult cohort with a CD prevalence of $3.3 \% .{ }^{11}$ More recently, Fuchs et al demonstrated that a tTG titre threshold of $10 \times \mathrm{ULN}$ was accurate at identifying individuals with Marsh 3 lesions across adult cohorts with different pretest probabilities for CD. ${ }^{28}$ However, in this study, the low CD pretest probability cohort included only older ( $>50$ years) adults. ${ }^{28}$ Furthermore, the performance characteristics of the $10 \times \mathrm{ULN}$ tTG threshold in a more general clinical scenario have been estimated, but not studied. ${ }^{33}$

Therefore, to confirm and extend these findings, we next sought to determine the predictive capacity of the $10 \times \mathrm{ULN}$ threshold against the presence of Marsh 3 lesions on duodenal biopsy in a cohort of individuals considered as low suspicion for CD (cohort 2). Low suspicion was determined on the basis that, in those referred for upper GI endoscopy, the referral bias for $C D$ was removed by excluding those who tested positive for $\mathrm{CD}$ in primary care. Indeed, the prevalence of CD in this population was $3.2 \%$, which aligns with the local prevalence of CD in symptomatic adults in primary care (3.6\%). ${ }^{34}$ These results demonstrate that $\mathrm{tTG}$ titres of $\geq 10 \times \mathrm{ULN}$ are equally good at predicting Marsh 3 lesions in the setting of both low and high disease pretest probability/prevalence and complement previous work that this diagnostic pathway can be used in adults with different risk levels for CD. ${ }^{11}{ }^{28}$ Importantly, while these data support the use of the biopsy avoidance approach in adults, we still advocate that suspected cases with tTG titres exceeding the $10 \times$ threshold are referred to a gastroenterologist for assessment, rather than the diagnosis of CD being made in primary care. In addition, these data were generated in adult cohorts within secondary and tertiary care settings, and thus, these results do not apply to screening the general population for a diagnosis of CD. These are important considerations to ensure that patients receive the correct diagnosis and to prevent the inappropriate institution of a GFD, which has been raised as concerns regarding this approach by some. ${ }^{23} 35$

One of the major challenges in implementing this diagnostic pathway is the variability and lack of standardisation between tTG assays. ${ }^{36}$ The inability to compare tTG results accurately between different assays increases the chances of misdiagnosis if a uniform threshold is used. In adults, there is a lack of data evaluating this in a multicentre international setting. To address this, we evaluated the $10 \times$ ULN threshold across multiple sites/countries and multiple assays (cohort 3). The PPV of the $10 \times \mathrm{ULN}$ threshold at detecting Marsh 3 lesions dropped from nearly 99\% across cohorts 1 and 2 at our UK centre to $95.2 \%$ in a multicentre international setting. This raises the concern that sole reliance on this as a marker for $\mathrm{CD}$ will lead to a false-positive diagnosis in around 1 in 20 cases.

Across all cohorts, the diagnostic performance of this pathway is based strictly on the presence of Marsh 3 histology. The PPV in all three cohorts increased to $>99 \%$, where both Marsh 2 and Marsh 3 lesions are accepted as CD. It is widely accepted that in the setting of relevant symptoms/high baseline risk of CD, raised serological markers, HLA DQ2/8 positivity and/or response to a GFD, the diagnosis of CD can be established in the absence of villous effacement. Indeed, this situation may represent a falsenegative histology rather than a false-positive serology ${ }^{28}$ due to a variety of reasons, including incorrect histological interpretation, which can vary in 10\%-25\% of cases between independent evaluations ${ }^{67}$ and/or patchy disease. ${ }^{32}$ Additionally, individuals with symptomatic CD may present with subtle villous abnormalities that fall within the scope of microscopic enteritis, which may lead to diagnostic inaccuracy. ${ }^{37}$

In comparison with the datasets that have evaluated the tTG IgA cut-off at $\geq 10 \times$ ULN included in the latest paediatric ESPGHAN guidelines, 2/30 reported a PPV of $<95 \%$; $7 / 30 \mathrm{had}$ a PPV between 95\% and 99\%; and 21/30 reported a PPV of >99\% for detecting Marsh 3 lesions. ${ }^{9}$ In studies where Marsh 
2 or Marsh 3 duodenal lesions were accepted for a diagnosis of CD, the PPV was $>97 \%$ in all studies. ${ }^{9}$ Thus, based on the current study, the performance characteristics of this diagnostic pathway in adults with different risk levels for CD or in a multicentre international setting is at least comparable with the studies included within the paediatric guidelines.

The high tTG threshold recommended by the paediatric community in part is included to control for tTG assay variation. ${ }^{36}$ We found that for some tTG assays, a lower cut-off may retain diagnostic specificity but increase the sensitivity of this approach. In contrast, the $10 \times \mathrm{ULN}$ threshold may not eliminate all false-positive cases across all assays. Therefore, a uniform threshold may hinder the clinical impact and accuracy of the no-biopsy approach in adult patients. Other studies have reported similar findings of differences in the optimal tTG thresholds and performance characteristics of different assays. ${ }^{14} 1928$ This does not imply that one assay is inferior to the other nor that using tTG threshold values in this manner is not appropriate, rather that the threshold ULN cut-off should be specific to the tTG assay, as there is currently no way to accurately standardise tTG results across different assays. ${ }^{38}$ Therefore, local validation of assay specific thresholds and/or limiting the use of this pathway to certain tTG assays may help to optimise the clinical impact and accuracy of this approach.

The latest ESPGHAN guidelines no longer consider either the presence of symptoms or HLA typing as mandatory in this diagnostic pathway. ${ }^{9}$ HLA typing has been shown to add no diagnostic value in this pathway in children or adults, and testing is costly and not widely available. ${ }^{928}$ Therefore, the HLA status of individuals was not specifically evaluated in this study. In addition, while each cohort comprised predominantly symptomatic adults, we did not specifically evaluate symptomatology in this study. Current ESPGHAN guidelines recommend confirming tTG titres with EMA positivity in a second blood sample. ${ }^{9}$ EMA status was not evaluated in all patients in the present study. While EMA positivity has been shown to enhance the predictive capacity of the biopsy avoidance approach, EMA testing incurs a high cost and is labour intensive, and the interpretation is subjective. ${ }^{35}$ For these reasons, some centres have stopped performing EMA tests. ${ }^{17}$ Therefore, including EMA testing in this algorithm may lead to poor uptake of this pathway in some areas. Alternative strategies have been suggested, including simultaneous tTG measurements ${ }^{28}$ or testing for deamidated gliadin peptides (DGPs) alongside tTG titres, the latter of which has shown promise in both adult and paediatric cohorts. ${ }^{1139}$ Further adult studies are awaited to address the place for DGPs in this algorithm.

Finally, it is noteworthy that the applicability of the biopsy avoidance approach is not as straightforward in adults as it is in children. Indeed, it should be stressed there are a number of reasons that a biopsy should still be performed in adults, particularly if there are 'red flag' signs/symptoms, such as, but not limited to, persistent dyspepsia in those aged $\geq 55$ years, weight loss, severe malnutrition, dysphagia and unexplained iron deficiency anaemia. ${ }^{40-42}$ Some of these presentations align with those of adult $\mathrm{CD}$; this underscores the importance of assessment by a gastroenterologist before a diagnosis of $\mathrm{CD}$ is made in the absence of a duodenal biopsy. In addition, a biopsy should also be considered in cases of discordance between serology and risk, and in borderline cases. ${ }^{27}$ Moreover, individuals with persistent symptoms despite adherence to a GFD require duodenal biopsy sampling, as tTG titres provide no information regarding histological improvement after introduction of a GFD. ${ }^{43}$
In conclusion, our data show that anti-tTG titres of $\geq 10 \times \mathrm{ULN}$ perform well at identifying adults with intestinal changes associated with CD. This study supports a no-biopsy strategy in adult gastroenterology services. This approach has implications in reducing the cost, risk and caseload associated with diagnostic endoscopy in adult CD. However, local validation of assay-specific thresholds would enable this approach to have the greatest impact on adult patients.

\section{Author affiliations}

${ }^{1}$ Academic Unit of Gastroenterology, Royal Hallamshire Hospital, Sheffield, UK 'Medicine, Gastroenterology Hospital 'Dr C Bonorino Udaondo', Buenos Aires, Argentina

${ }^{3}$ Gastroenterology \& Hepatology Section, Department of Medicine, University of Perugia Medical School, Perugia, Italy

${ }^{4}$ Department Clinical Chemistry, Amsterdam Gastroenterology and Metabolism and Infection and Immunity Institutes, Amsterdam UMC, Amsterdam, The

Netherlands

${ }^{5}$ Department of Health Promotion Sciences, Maternal and Infant Care, Internal Medicine and Medical Specialties (PROMISE), University of Palermo, Palermo, Italy ${ }^{6}$ Pathology Department, Grigore T. Popa University of Medicine and Pharmacy lasi, Iasi, Romania

${ }^{7}$ Cardiovascular and Medical Sciences, University of Glasgow, Glasgow, UK

${ }^{8}$ Department of Pathology, Ankara University Medical School, Ankara, Turkey ${ }^{9}$ Department of Gastroenterology and Hepatology, Faculty of Medicine, Mashhad University of Medical Sciences, Mashhad, Iran

${ }^{10}$ Celiac Disease Center, Department of Medicine, Columbia University College of Physicians and Surgeons, New York, New York, USA

${ }^{11}$ Gastroenterology, Luton and Dunstable Hospital NHS Foundation Trust, Luton, UK

${ }^{12}$ Department of Gastroenterology, Dudley Group NHS Foundation Trust, Birmingham City University, Birmingham, UK

${ }^{13}$ Gastroenterology Department, Grigore T. Popa University of Medicine and Pharmacy lasi, lasi, Romania

${ }^{14}$ Gastroenterology and Liver Diseases Research Center, Research Institute for Gastroenterology and Liver Diseases, Shahid Beheshti University of Medical Sciences, Tehran, Iran

${ }^{15}$ Department of Gastroenterology, Queen Elizabeth II Hospital, Hertfordshire, UK

${ }^{16}$ Pathology, Brigham and Women's Hospital, Boston, Massachusetts, USA

${ }^{17}$ Department of Medical and Surgical Sciences, University of Bologna, Bologna, Italy

${ }^{18}$ Department of Pathology, Spedali Civili, Brescia, Italy

${ }^{19}$ Department of Gastroenterology, MidCentral District Health Board, Palmerston North, New Zealand

Contributors HAP analysed the data and wrote the original manuscript. KR and DSS conceived the study, collected the data and edited the manuscript. SAR, ML, LJSM and EMRB recruited patients and helped collect and collate the data. JB, GB, HJB, AC, MD, MHD, AE, AG, PHRG, MWJ, SI, BL, AL, RM, HMS, MR-N, DR, IS, AS, UV, VV, GW and SC helped collect/generate the data. All authors approved the final version of the manuscript.

Funding BL is supported by The Louis and Gloria Flanzer Philanthropic Trust.

Competing interests DSS receives an educational grant from Dr Schär (a gluten-free food manufacturer). PHRG serves on the advisory board of ImmusanT, Cellimmune and ImmunogenX, and is an unpaid member of Nima's Scientific Advisory Board. The remaining authors disclose no conflicts.

Patient and public involvement Patients and/or the public were not involved in the design, conduct, reporting or dissemination plans of this research.

\section{Patient consent for publication Not required.}

Ethics approval The Sheffield arm of the study (cohorts 1 and 2) was approved by the Yorkshire and the Humber Sheffield Research Ethics Committee, under registration numbers $14 / \mathrm{YH} / 1216$ and STH15416, respectively. Data relating to the multicentre cohort were previously approved by the research and development/audit departments of countries involved and were fully approved by the ethical committee of the Research Institute for Gastroenterology and Liver Disease, Shahid Beheshti University of Medical Science Tehran (protocol IR.SBMU.RIGLD.REC.1395.87) or the Columbia University Irving Medical Center Institutional Review Board (protocol IRB-AAAB0960)

Provenance and peer review Not commissioned; externally peer reviewed.

Data availability statement No data are available.

Open access This is an open access article distributed in accordance with the Creative Commons Attribution Non Commercial (CC BY-NC 4.0) license, which permits others to distribute, remix, adapt, build upon this work non-commercially, and license their derivative works on different terms, provided the original work is 
properly cited, appropriate credit is given, any changes made indicated, and the use is non-commercial. See: http://creativecommons.org/licenses/by-nc/4.0/.

\section{ORCID iDs}

Hugo A Penny http://orcid.org/0000-0003-1610-2282

Antonio Carroccio http://orcid.org/0000-0001-8913-7916

Sauid Ishaq http://orcid.org/0000-0003-1458-4708

Amitabh Srivastava http://orcid.org/0000-0001-5165-8142

\section{REFERENCES}

1 Lebwohl B, Sanders DS, Green PHR. Coeliac disease. Lancet 2018;391:70-81.

2 Singh P, Arora A, Strand TA, et al. Global Prevalence of Celiac Disease: Systematic Review and Meta-analysis. Clin Gastroenterol Hepatol 2018;16:823-36.

3 Marsh MN. Gluten, major histocompatibility complex, and the small intestine. A molecular and immunobiologic approach to the spectrum of gluten sensitivity ('celiac sprue'). Gastroenterology 1992;102:330-54.

4 Tierney M, Bevan R, Rees CJ, et al. What do patients want from their endoscopy experience? The importance of measuring and understanding patient attitudes to their care. Frontline Gastroenterol 2016;7:191-8.

5 Thompson AM, Wright DJ, Murray W, et al. Analysis of 153 deaths after upper gastrointestinal endoscopy: room for improvement? Surg Endosc 2004;18:22-5

6 Arguelles-Grande C, Tennyson CA, Lewis SK, et al. Variability in small bowel histopathology reporting between different pathology practice settings: impact on the diagnosis of coeliac disease. J Clin Pathol 2012;65:242-7.

7 Mubarak A, Nikkels P, Houwen R, et al. Reproducibility of the histological diagnosis of celiac disease. Scand J Gastroenterol 2011;46:1065-73.

8 Husby S, Koletzko S, Korponay-Szabó IR, et al. European Society for pediatric gastroenterology, hepatology, and nutrition guidelines for the diagnosis of coeliac disease. J Pediatr Gastroenterol Nutr 2012;54:136-60.

9 Husby S, Koletzko S, Korponay-Szabó I, et al. European Society paediatric gastroenterology, hepatology and nutrition guidelines for diagnosing coeliac disease 2020. J Pediatr Gastroenterol Nutr 2020;70:141-56.

10 Hill PG, Holmes GKT. Coeliac disease: a biopsy is not always necessary for diagnosis. Aliment Pharmacol Ther 2008;27:572-7.

11 Sugai E, Moreno ML, Hwang HJ, et al. Celiac disease serology in patients with different pretest probabilities: is biopsy avoidable? World I Gastroenterol 2010;16:3144-52

12 Zanini B, Magni A, Caselani F, et al. High tissue-transglutaminase antibody level predicts small intestinal villous atrophy in adult patients at high risk of celiac disease. Dig Liver Dis 2012;44:280-5.

13 Alessio MG, Tonutti E, Brusca I, et al. Correlation between IgA tissue transglutaminase antibody ratio and histological finding in celiac disease. J Pediatr Gastroenterol Nutr 2012;55:44-9.

14 Beltran L, Koenig M, Egner W, et al. High-titre circulating tissue transglutaminase-2 antibodies predict small bowel villous atrophy, but decision cut-off limits must be locally validated. Clin Exp Immunol 2014;176:190-8.

15 Wakim-Fleming Jamile', Pagadala MR, Lemyre MS, et al. Diagnosis of celiac disease in adults based on serology test results, without small-bowel biopsy. Clin Gastroenterol Hepatol 2013;11:511-6.

16 Tortora R, Imperatore N, Capone P, et al. The presence of anti-endomysial antibodies and the level of anti-tissue transglutaminases can be used to diagnose adult coeliac disease without duodenal biopsy. Aliment Pharmacol Ther 2014;40:1223-9.

17 Oyaert M, Vermeersch P, De Hertogh G, et al. Combining antibody tests and taking into account antibody levels improves serologic diagnosis of celiac disease. Clin Chem Lab Med 2015:53:1537-46.

18 Holmes GKT, Forsyth JM, Knowles S, et al. Coeliac disease: further evidence that biopsy is not always necessary for diagnosis. Eur J Gastroenterol Hepatol 2017;29:640-5

19 Efthymakis K, Serio M, Milano A, et al. Application of the Biopsy-Sparing ESPGHAN guidelines for celiac disease diagnosis in adults: a real-life study. Dig Dis Sci 2017;62:2433-9.
20 Previtali G, Licini L, D'Antiga L, et al. Celiac disease diagnosis without biopsy: is a 10x ULN Antitransglutaminase result suitable for a chemiluminescence method? J Pediatr Gastroenterol Nutr 2018;66:645-50.

21 Ganji A, Esmaeilzadeh A, Bahari A, et al. Correlation between cut-off level of tissue transglutaminase antibody and marsh classification. Middle East J Dig Dis 2016;8:318-22.

22 Working group set up by the Finnish Medical Society Duodecim and the Finnish Gastroenterology Society, Celiac disease. Current care guidelines, 2018, 2020. Available: http://www.kaypahoito.fi/web/kh/suositukset/suositus?id=hoi08001\#K1

23 Marks LJ, Kurien M, Sanders DS. The serological diagnosis of adult coeliac disease - a cautious step forward? Gastroenterol Hepatol Bed Bench 2018;11:175-7.

24 Lau MS, Mooney PD, White WL, et al. Office-Based point of care testing (IgA/ IgG-Deamidated gliadin peptide) for celiac disease. Am J Gastroenterol 2018;113:1238-46.

25 Marsh MN, Rostami K. What is a normal intestinal mucosa? Gastroenterology 2016;151:784-8

26 Ludvigsson JF, Bai JC, Biagi F, et al. Diagnosis and management of adult coeliac disease: guidelines from the British Society of gastroenterology. Gut 2014;63:1210-28.

27 Bai JC, Verdú EF. Evolving paradigms in the diagnosis of adult patients with celiac disease. Am J Gastroenterol 2019;114:854-7.

28 Fuchs V, Kurppa K, Huhtala H, et al. Serology-based criteria for adult coeliac disease have excellent accuracy across the range of pre-test probabilities. Aliment Pharmacol Ther 2018;00:1-8.

29 Mooney PD, Kurien M, Evans KE, et al. Clinical and immunologic features of ultrashort celiac disease. Gastroenterology 2016;150:1125-34.

30 Hopper AD, Cross SS, Hurlstone DP, et al. Pre-endoscopy serological testing for coeliac disease: evaluation of a clinical decision tool. BMJ 2007:334:729.

31 Evans KE, Aziz I, Cross SS, et al. A prospective study of duodenal bulb biopsy in newly diagnosed and established adult celiac disease. Am J Gastroenterol 2011;106:1837-1742.

32 Hopper AD, Cross SS, Sanders DS. Patchy villous atrophy in adult patients with suspected gluten-sensitive enteropathy: is a multiple duodenal biopsy strategy appropriate? Endoscopy 2008;40:219-24.

33 Fernández-Bañares F, Alsina M, Modolell I, et al. Are positive serum-IgA-tissuetransglutaminase antibodies enough to diagnose coeliac disease without a small bowel biopsy? Post-test probability of coeliac disease. J Crohns Colitis 2012;6:861-6.

34 Sanders DS, Patel D, Stephenson TJ, et al. A primary care cross-sectional study of undiagnosed adult coeliac disease. Eur J Gastroenterol Hepatol 2003:15:407-13.

35 Penny HA, Raju SA, Sanders DS. Progress in the serology-based diagnosis and management of adult celiac disease. Expert Rev Gastroenterol Hepatol 2020;14:147-54.

36 Popp A, Kivelä L, Fuchs V, et al. Diagnosing celiac disease: towards Wide-Scale screening and serology-based criteria? Gastroenterol Res Pract 2019;2019:2916024.

37 Rostami K, Aldulaimi D, Holmes G, et al. Microscopic enteritis: Bucharest consensus. World J Gastroenterol 2015;21:2593-604.

38 Egner W, Shrimpton A, Sargur R, et al. ESPGHAN guidance on coeliac disease 2012: multiples of ULN for decision making do not harmonise assay performance across centres. J Pediatr Gastroenterol Nutr 2012;55:733-5.

39 Wolf J, Hasenclever D, Petroff D, et al. Antibodies in the diagnosis of coeliac disease: a Biopsy-Controlled, international, multicentre study of 376 children with coeliac disease and 695 controls. PLoS One 2014;9:e97853.

40 ASGE Standards of Practice Committee, Early DS, Ben-Menachem T, et al. Appropriate use of GI endoscopy. Gastrointest Endosc 2012;75:1127-31.

41 ASGE Standards of Practice Committee, Shaukat A, Wang A, et al. The role of endoscopy in dyspepsia. Gastrointest Endosc 2015;82:227-32.

42 Biagi F, Marchese A, Ferretti F, et al. A multicentre case control study on complicated coeliac disease: two different patterns of natural history, two different prognoses. BMC Gastroenterol 2014;14:139.

43 Silvester JA, Kurada S, Szwajcer A, et al. Tests for serum transglutaminase and Endomysial antibodies do not detect most patients with celiac disease and persistent villous atrophy on gluten-free diets: a meta-analysis. Gastroenterology 2017:153:689-701. 